Pacific Journal of Mathematic 


\title{
A LIMIT-POINT CRITERION FOR EXPRESSIONS WITH OSCILLATORY COEFFICIENTS
}

\author{
THOMAS T. READ
}

A criterion is given for the equation $-\left(p y^{\prime}\right)^{\prime}+q y=0$ to have a solution on the interval $[a, \infty)$ which is not in $L_{2}(a, \infty)$. The criterion permits $q$ (or $\operatorname{Re} q$ if $q$ is complexvalued) to be decomposable into a sum $q=q_{1}+q_{2}$ where the expression $-\left(p y^{\prime}\right)^{\prime}+q_{1} y$ essentially satisfies the well-known limit-point criterion of Levinson and $q_{2}$ may be thought of as an oscillatory function whose amplitude may be large, but whose integral over $[a, x]$ increases relatively slowly as a function of $x$.

1. Introduction. Let $p$ be a positive function and let $q$ be a complex-valued function on the interval $[a, \infty), a>-\infty$, such that $1 / p$ and $q$ are Lebesgue integrable on each finite interval $[a, b]$. Let $M$ be the differential expression

$$
M(y)=-\left(p y^{\prime}\right)^{\prime}+q y .
$$

Then we shall obtain conditions on $p$ and $q$ sufficient for the equation $M(y)=0$ to have a solution which is not in $L_{2}(a, \infty)$.

The conditions, stated precisely in Theorem 1 at the beginning of $\S 2$, place no restriction on the imaginary part of $q$. For real-valued $q$ they extend the well-known limit point criterion of Levinson [8]. (See also Coddington and Levinson [3; page 229].) Roughly, for a given $p$, Levinson's condition limits the rate at which $q(x)$ can approach $-\infty$ as $x \rightarrow \infty$. Theorem 1 extends this by allowing $q$ to be decomposable into a sum $q=q_{1}+q_{2}$ where $q_{1}$ satisfies a condition very similar to Levinson's, and where the integral of $q_{2}$ over $[a, x]$ grows relatively slowly as a function of $x$.

A simple example of such an expression is

$$
-\left(x y^{\prime}\right)^{\prime}-\left(x+x e^{x} \sin \left(e^{x}\right)\right) y .
$$

A slightly more complicated one is

$$
-y^{\prime \prime}-\left(x+x^{3}(\sin x)^{4}+x^{5} \sin \left(x^{6}\right)\right) y \text {. }
$$

We shall return to these examples in $\$ 3$.

Another very well-known limit-point criterion, due to Hartman and Wintner [5], states for $p=1$ and $q$ real-valued that if the negative part, 
$q^{-}$, of $q$ satisfies $\int_{a}^{x} q^{-} d t \leqq K x^{3}$ for all sufficiently large $x$, then (1.1) is limit-point. Their proof, which relies on an analysis of the frequency of the zeros of solutions of the equation $M(y)=0$, has been refined by Eastham [4] to yield a criterion dependent only on estimates of the integral of $q^{-}$over a suitable sequence of pairwise disjoint intervals. We shall show in $\S 4$ that this interval criterion is a consequence of Theorem 1 by deriving an interval criterion for (1.1) that reduces to Eastham's result for $p=1$.

For $p=1$ and real-valued $q$ one form of our result is essentially equivalent to a recent limit-point criterion of Knowles [7]. This equivalence, which is not entirely obvious, is demonstrated in $\$ 5$.

Theorem 1 resembles Theorem 1 of Atkinson and Evans [1] in permitting complex-valued $q$ and also the possibility of considering square-integrability with respect to a weight function. However, the conditions on $\operatorname{Re} q$ in the two theorems are independent.

Finally we note that the hypotheses of our theorem are largely invariant under the addition to (1.1) of a term $Q y$ where the integral of $Q$ is small in a suitable sense. We illustrate this in $\$ 6$ with an extension of a theorem of Patula and Wong [9].

\section{The main result.}

THEOREM 1. Suppose that there exists a nonnegative locally absolutely continuous function $w$ on $[a, \infty)$ such that

(i) $p w^{\prime 2} \leqq K_{1}^{2}$ a.e.,

(ii) $\int_{a}^{\infty} w p^{-1 / 2} d t=\infty$

$\operatorname{Re} q=q_{1}+q_{2}$ where

(iii) $-q_{1} w^{2} \leqq K_{2}$,

(iv) $\left|\int_{a}^{x} q_{2} w^{1-b} d t\right|\{w(x)\}^{b}\{p(x)\}^{-1 / 2} \leqq K_{3}$ for some $b, 0 \leqq b \leqq 1$ and all $x \geqq a$.

Then the equation $-\left(p y^{\prime}\right)^{\prime}+q y=0$ has a solution which is not of integrable square on $[a, \infty)$.

Remarks. 1. If $q=q_{1}$ is real-valued, $q_{2}=0$, and if $w$ is positive, then the change of notation $M=w^{-2}$ gives Levinson's limit-point criterion as stated in [3; page 229].

2. In general, if $q$ is real-valued, then the conclusion of Theorem 1 may be rephrased to the statement that the expression (1.1) is limitpoint. When $q$ is complex-valued this may still be done in some circumstances (for instance if $\operatorname{Im} q$ is semi-bounded) provided the definition of the limit-point condition given in Kauffman [6] is used. 
3. If $k$ is a positive locally integrable function which is not in $L_{1}(a, \infty)$, then Theorem 1 can easily be adapted to a criterion for the equation $-\left(p y^{\prime}\right)^{\prime}+q y=0$ to possess a solution $y$ such that $\int_{a}^{\infty}|y|^{2} k d t=$ $\infty$. The right sides of (i), (iii), and (iv) then become $K_{1}^{2} k, K_{2} k$, and $K_{3} k^{1 / 2}$ respectively and (ii) becomes $\int_{a}^{\infty} w(k / p)^{1 / 2} d t=\infty$. The manner in which the weight function enters (i), (ii), and (iii) is the same as in Theorem 1 of [1]. We shall comment at the end of the proof of Theorem 1 on the small alterations necessary to deal with this version.

Proof. Let $y$ and $z$ be solutions of $M(y)=0$ such that

$$
p\left(y^{\prime} z-y z^{\prime}\right)=1 \text {. }
$$

Set

$$
r=\left(|y|^{2}+|z|^{2}\right)^{1 / 2}
$$

We must show that $r$ is not of integrable square. A straightforward calculation establishes that $r$ satisfies the equation

$$
\left(p r^{\prime}\right)^{\prime}-(\operatorname{Re} q) r=S
$$

where

$$
S=\left(p / r^{3}\right)\left[\left(\left|y^{\prime}\right|^{2}+\left|z^{\prime}\right|^{2}\right)\left(|y|^{2}+|z|^{2}\right)-\left(\operatorname{Re}\left(y^{\prime} \bar{y}+z^{\prime} \bar{z}\right)\right)^{2}\right] .
$$

We assert that $S \geqq 1 / 2 p r^{3}$. To see this, let $u_{1}, u_{2}, u_{3}, u_{4}$ be real-valued functions such that $y=u_{1}+i u_{2}$, and $z=u_{3}+i u_{4}$. Then

$$
S=\left(p / r^{3}\right) \sum_{j<k}\left(u_{j}^{\prime} u_{k}-u_{\jmath} u_{k}^{\prime}\right)^{2}
$$

The real part of (2.1) may be written as

$$
p\left[\left(u_{1}^{\prime} u_{3}-u_{1} u_{3}^{\prime}\right)-\left(u_{2}^{\prime} u_{4}-u_{2} u_{4}^{\prime}\right)\right]=1
$$

Thus,

$$
\begin{aligned}
S & \geqq\left(p / r^{3}\right)\left[\left(u_{1}^{\prime} u_{3}-u_{1} u_{3}^{\prime}\right)^{2}+\left(u_{2}^{\prime} u_{4}-u_{2} u_{4}^{\prime}\right)^{2}\right] \\
& \geqq\left(p / r^{3}\right)\left(1 / 2 p^{2}\right)=1 / 2 p r^{3} .
\end{aligned}
$$

Now let $w$ be as in the hypotheses of Theorem 1 . By inserting the estimate just obtained for $S$ into (2.2), multiplying by $r w^{2}$, and integrating, we obtain that for each positive $x$, 


$$
\begin{gathered}
\int_{a}^{x}\left(p r^{\prime}\right)^{\prime} r w^{2} d t-\int_{a}^{x} q_{1} r^{2} w^{2} d t-\int_{a}^{x} q_{2} r^{2} w^{2} d t \\
\geqq \int_{a}^{x} w^{2} / 2 p r^{2} d t .
\end{gathered}
$$

Suppose that $\|r\|^{2}=\int_{a}^{\infty} r^{2} d t$ is finite. Then by Schwarz's inequality,

$$
\int_{a}^{x} w^{2} / 2 p r^{2} d t \geqq\left(1 / 2\|r\|^{2}\right)\left(\int_{a}^{x} w p^{-1 / 2} d t\right)^{2}
$$

Hence, by (ii), $\int_{a}^{x} w^{2} / 2 p r^{2} d t \rightarrow \infty$ as $x \rightarrow \infty$. We shall see that this leads to a contradiction.

Set $H(x)=\int_{a}^{x} p\left(r^{\prime}\right)^{2} w^{2} d t$. An integration by parts yields that

$$
\left.\int_{a}^{x}\left(p r^{\prime}\right)^{\prime} r w^{2} d t=p r r^{\prime} w^{2}\right]_{a}^{x}-H(x)-2 \int_{a}^{x} p r r^{\prime} w w^{\prime} d t
$$

By (i) and Schwarz's inequality again,

$$
\int_{a}^{x} p r r^{\prime} w w^{\prime} d t \leqq K_{1}\|r\|\{H(x)\}^{1 / 2}
$$

From another integration by parts,

$$
\int_{a}^{x} q_{2} r^{2} w^{2} d t=\{r(x)\}^{2}\{w(x)\}^{1+b} \int_{a}^{x} q_{2} w^{1-b} d t-I(x)
$$

where

$$
I(x)=\int_{a}^{x}\left(\int_{a}^{t} q_{2} w^{1-b} d s\right)\left[2 r r^{\prime} w^{1+b}+(1+b) r^{2} w^{b} w^{\prime}\right] d t
$$

Now

$$
\begin{aligned}
|I(x)| & \leqq 2 K_{3} \int_{a}^{x} p^{1 / 2} r r^{\prime} w d t+(1+b) K_{3} \int_{a}^{x} p^{1 / 2} r^{2} w^{\prime} d t \\
& \leqq 2 K_{3}\|r\|\{H(x)\}^{1 / 2}+(1+b) K_{1} K_{3}\|r\|^{2}
\end{aligned}
$$

The other term on the right side of (2.4) satisfies

$$
\{r(x)\}^{2}\{w(x)\}^{1+b}\left|\int_{a}^{x} q_{2} w^{1-b} d t\right| \leqq K_{3}\{r(x)\}^{2} w(x)\{p(x)\}^{1 / 2}
$$

Finally, $\int_{a}^{x} q_{1} r^{2} w^{2} d t \leqq K_{2}\|r\|^{2}$. 
Thus (2.3) may be rewritten in the form

$$
A(x)-\left[H(x)-K_{4}\{H(x)\}^{1 / 2}-K_{5}\right] \geqq \int_{a}^{x} w^{2} / 2 p r^{2} d t
$$

where $K_{4}$ and $K_{5}$ are positive constants and

$$
A=p w^{2} r r^{\prime}+K_{3} p^{1 / 2} w r^{2} .
$$

The expression in brackets on the left side of (2.5) either is bounded (if $H$ is) or approaches $+\infty$ as $x \rightarrow \infty$ (if $H$ does). In either case we must have that $A(x) \rightarrow+\infty$ as $x \rightarrow \infty$. We complete the proof by showing that this cannot occur.

Set $f=p^{1 / 2} w$ and $g=r^{2}$. Then $2 A=f^{2} g^{\prime}+2 K_{3} f g$. Note that $g$ is in $L_{1}(a, \infty)$. We now use this decomposition of $A$ to show that $A$ cannot even be bounded away from 0 on an interval $[d, \infty)$. For suppose that $A(x) \geqq c>0$ for $x \geqq d$. Set $N=\left\{x \geqq d: g^{\prime}(x)<0\right\}$. Then on $N$, $2 K_{3} f g>\left|f^{2} g^{\prime}\right|$ and also $K_{3} f g>c$ so that $\left(K_{3} / c\right) g>1 / f$. Combining these yields that

$$
\int_{N}-g^{\prime} / g d t<\left(2 K_{3}^{2} / c\right) \int_{N} g d t<\infty
$$

Hence $\log (g(x) / g(d))>-(1 / 2 c) \int_{N} g d t$ so that $g$ is bounded away from 0 . But this is impossible since $g$ is in $L_{1}(a, \infty)$. Thus $A$ cannot be bounded away from 0 and, in particular, cannot satisfy (2.5) for all positive $x$. Thus it must be the case that $r$ is not of integrable square and the proof is complete.

We now comment briefly on the changes necessary to incorporate a weight function $k$ as mentioned in Remark 3 above. Inequality (2.5) can be derived essentially as before under the assumption that $\int_{a} r^{2} k d t$ is finite, where now $2 A=f^{2} g^{\prime}+2 K_{3} f g k^{1 / 2}$ ( $f$ and $g$ are as before). Then $A(x) \geqq c$ for $x \geqq d$ implies that on $N=\left\{x \geqq d: g^{\prime}(x)<0\right\},\left(2 K_{3}^{2} / c\right) g k>$ $-g^{\prime} / g$ and one again has the contradiction that $g$ is bounded away from 0 . On the other hand it follows as before from Schwarz's inequality and the new form of (ii) that the right side of (2.5) approaches $\infty$ as $x \rightarrow \infty$. Thus again (2.5) cannot hold for all positive $x$ and the conclusion follows as before.

3. Examples. In this section we return to the examples (1.2) and (1.3) mentioned in the introduction. For (1.2), set $q_{1}(x)=x, q_{2}(x)=$ 
$x e^{x} \sin \left(e^{x}\right)$, and $w(x)=x^{-1 / 2}$. Then $w(x) p(x)^{-1 / 2}=x^{-1}$ and $\int_{1}^{x} q_{2} d t=$ $-x \cos \left(e^{x}\right)+\cos e$. Thus the hypotheses of Theorem 1 are clearly satisfied on $[1, \infty)$ with $b=1$. Note that with the same simple choice for $w$ one could substitute $q_{2}(x)=x^{d} \sin \left(x^{d}\right)$, since it is easy to see that then $\left|\int_{1}^{x} q_{2} d t\right| \leqq K x$.

To deal with (1.3) we must choose $w$ more carefully. Here we take $q_{1}(x)=x+x^{3}(\sin x)^{4}$, and $q_{2}(x)=x^{5} \sin \left(x^{6}\right)$. This example, with $q=q_{1}$, has been discussed by Eastham [4]. Inspired by his discussion we set $I_{n}=\left[n \pi-n^{-1 / 2}, n \pi+n^{-1 / 2}\right], n=1,2, \cdots$. Define $w$ on $I_{n}$ by $w(x)=$ $x-n \pi+n^{-1 / 2}$ for $n \pi-n^{-1 / 2} \leqq x \leqq n \pi$ and $w(x)=w(2 n \pi-x)$ on the rest of $I_{n}$. On the complement of the union of the $I_{n}$ 's define $w(x)=$ 0 . On $I_{n}, w(x) \leqq n^{-1 / 2}, q_{1}(x) \leqq K n$, and $\left|\int_{0}^{x} q_{2} d t\right| \leqq 1 / 6$. Thus (i), (iii), and (iv) are satisfied. Finally, $\int_{I_{n}} w d t=1 / n$ so (ii) is also satisfied and it follows from Theorem 1 that (1.3) is limit-point.

4. An interval criterion. We shall now establish a limitpoint criterion for (1.1) (with $q$ real-valued) in which the coefficients are restricted only on a sequence of pairwise disjoint intervals. The argument is a refinement of that used in the previous section to treat (1.3). For $p=1$ the result is due to Eastham [4] by a quite different method. Thus Theorem 1 may be regarded as a step toward the integration of the "interval criteria" and the criteria of Levinson type into a common theory.

THEOREM 2. Suppose that there is a sequence $\left\{I_{n}\right\}_{n=1}^{\infty}, I_{n}=\left[a_{n}, b_{n}\right]$, of pairwise disjoint intervals in $[a, \infty)$ and a sequence $\left\{v_{n}\right\}_{n=1}^{\infty}$ of positive numbers such that for each $n$,

(ii) $\sum_{n=1}^{\infty} v_{n}^{-1}=\infty$,

(iii) $\int_{a_{n}}^{b_{n}} q^{-} d t \leqq C v_{n}^{2} P_{n}^{3} \min _{I_{n}} p^{1 / 2}$ where $q^{-}$is the negative part of $q$.

Then $-(p y)^{\prime}+q y$ is limit-point.

Proof. We may assume that $K \geqq 1$. Divide each $I_{n}$ into $\left[v_{n} P_{n}^{2}\right]+1$ subintervals $([\cdots]$ is the greatest integer function) so that on each subinterval $J$,

$$
\left(2 v_{n} P_{n}\right)^{-1} \leqq \int_{J} p^{-1 / 2} d t \leqq\left(v_{n} P_{n}\right)^{-1} .
$$

On at least half of these, 


$$
\int_{J} q^{-} d t \leqq 2 C v_{n} P_{n} \min _{I_{n}} p^{1 / 2}
$$

For each subinterval $J=[c, d] \subset I_{n}$ on which (4.1) is valid, choose $e \in(c, d)$ so that

$$
\int_{c}^{e} p^{-1 / 2} d t=\left(\int_{J} p^{-1 / 2} d t\right) / 2
$$

Define $w$ on each such subinterval by $w(t)=\int_{c}^{t} p^{-1 / 2} d t$ for $c \leqq t \leqq e$ and $w(t)=w(e)-\int_{e}^{t} p^{-1 / 2} d t$ for $e \leqq t \leqq d$. Then on $J$,

$$
\max w=(1 / 2) \int_{J} p^{-1 / 2} d t \leqq\left(2 v_{n} P_{n}\right)^{-1}
$$

and

$$
\int_{J} w p^{-1 / 2} d t=(1 / 4)\left(\int_{J} p^{-1 / 2} d t\right)^{2} \geqq\left(4 v_{n} P_{n}\right)^{-2}
$$

Set $w=0$ on the remaining subintervals of each $I_{n}$ and on the complement of $\cup I_{n}$.

Then for each $n$, since (4.1) holds on at least $(1 / 2)\left(\left[v_{n} P_{n}^{2}\right]+1\right)$ subintervals of $I_{n}$,

$$
\int_{I_{n}} w p^{-1 / 2} d t \geqq\left(4 v_{n} P_{n}\right)^{-2}(1 / 2) v_{n} P_{n}^{2}=(1 / 32) v_{n}^{-1}
$$

Hence by (ii), $\int_{a}^{\infty} w p^{-1 / 2} d t=\infty$. Also $w$ is clearly absolutely continuous with $p\left(w^{\prime}\right)^{2} \leqq 1$ a.e.. Thus (i) and (ii) of Theorem 1 are satisfied. It remains to construct a suitable decomposition of $q$.

Define a step function $q_{0}$ which is constant on each of the subintervals $J=[c, d]$ constructed above (whether (4.1) holds or not) by

$$
q_{0}=\int_{c}^{d} q^{-} d t /(d-c)
$$

on $[c, d]$.

Similarly, on the interval $\left[b_{n}, a_{n+1}\right]$ between $I_{n}$ and $I_{n+1}$, set $q_{0}=$ $\int_{b_{n}}^{a_{n+1}} q^{-} d t /\left(a_{n+1}-b_{n}\right)$. Thus $[a, \infty)$ is the union of subintervals on each of which $\int\left(q^{-}-q_{0}\right) d t=0$. Note that $q_{0} w^{2}$ is bounded above on 
$[a, \infty)$. This is clear outside the union of the subintervals where (4.1) holds, for then $w=0$. On a subinterval $J=[c, d] \subset I_{n}$ for which (4.1) is valid,

$$
q_{0} w^{2} \leqq 2 C v_{n} P_{n} \min _{I_{n}} p^{1 / 2} /\left(2 v_{n} P_{n}\right)^{2}(d-c) \leqq C
$$

since $\min p^{1 / 2} /(d-c) \leqq\left(\int_{J} p^{-1 / 2}\right)^{-1} \leqq 2 v_{n} P_{n}$.

Now decompose $q$ by setting $q_{1}=q^{+}-q_{0}$, and $q_{2}=-q^{-}+q_{0}$. From the previous paragraph, $-q_{1} w^{2} \leqq C$ on $[a, \infty)$. Also $w p^{-1 / 2}(x) \int_{a}^{x} q_{2} d t$ is nonzero only in the subintervals on which (4.1) holds, and on such a subinterval $[c, d], \int_{a}^{x} q_{2} d t=\int_{c}^{x} q_{2} d t$ since $\int_{a}^{c} q_{2} d t=0$. Hence on such a subinterval

$$
w p^{-1 / 2}(x)\left|\int_{a}^{x} q_{2} d t\right| \leqq 2 C v_{n} P_{n} \min _{I_{n}} p^{1 / 2} / 2 v_{n} P_{n} p^{1 / 2}(x) \leqq C .
$$

Thus (iii) and (iv) of Theorem 1 are also satisfied for this decomposition and Theorem 2 now follows from that result.

5. A theorem of Knowles. In this section we shall derive from Theorem 1 the following result of Knowles [7] which was established by a refinement of a method due to Brinck [2]. Note that $w$ is required here to be strictly positive (because of (iii)) rather than nonnegative as in Theorem 1.

THEOREM 3. Suppose that there is a positive locally absolutely continuous function $w$ such that

(i) $w^{\prime}$ is bounded a.e.,

(ii) $\int_{a}^{\infty} w d t=\infty$

(iii) there exists a constant $C$ such that $-\int_{I} q w d t \leqq C$ for each interval I for which $\int_{I} w^{-1} d t \leqq 1$.

Then $-y^{\prime \prime}+q y$ is limit-point.

Proof. Hypotheses (i) and (ii) are identical to those of Theorem 1 for $p=1$. It remains to consider (iii). For this we need the equivalence described in the following lemma. The author is grateful to Professor W. N. Everitt for bringing this equivalence to his attention. 
LemMA. Let $\mu$ be a positive continuous regular Borel measure on $[a, \infty)$, and let $Q$ be a real-valued function defined on this interval. Then the following properties are equivalent.

(i) There is a constant $C$ such that $-\int_{I} Q d \mu \leqq C$ for each interval I for which $\mu(I) \leqq 1$.

(ii) $Q=Q_{1}+Q_{2}$ where $-Q_{1} \leqq C_{1}$ and $\left|\int_{a}^{x} Q_{2} d \mu\right| \leqq C_{2}$ for all $x \geqq a$.

Proof of Lemma. It is clear that (ii) implies (i) with $C=C_{1}+2 C_{2}$ since for any interval $[c, d]$,

$$
-\int_{c}^{d} Q_{2} d \mu=\int_{a}^{c} Q_{2} d \mu-\int_{a}^{d} Q_{2} d \mu \leqq 2 C_{2} .
$$

For the other direction, set $x_{0}=a$ and for each $n$ choose $x_{n}$ so that $\mu\left(\left[x_{n-1}, x_{n}\right]\right)=1$. If $\mu\left(\left[x_{n-1}, \infty\right)\right)<1$, set $x_{n}=\infty$. The proof will not be affected by whether the sequence $\left\{x_{n}\right\}$ is finite or infinite. Suppose that on each $\left[x_{n-1}, x_{n}\right]$ we can write $Q=Q_{1}+Q_{2}$ where $-Q_{1} \leqq C$, $\left|\int_{a}^{x} Q_{2} d \mu\right| \leqq C$ for all $x_{n-1} \leqq x \leqq x_{n}$, and $\int_{x_{n-1}}^{x_{n}} Q_{2} d \mu=0$. Then, combining the decompositions, we will have that $-Q_{1} \leqq C$ on $[a, \infty)$ and that if $x_{n-1}<x \leqq x_{n}$, then

$$
\left|\int_{a}^{x} Q_{2} d \mu\right|=\left|\int_{x_{n-1}}^{x} Q_{2} d \mu\right| \leqq C
$$

Thus the lemma will be proved.

To simplify notation slightly we consider $\left[a, x_{1}\right]$. Let $\int_{a}^{x_{1}} Q d \mu=$ $\alpha C$. Note that (i) then implies that $\int_{a}^{x} Q d \mu \leqq(\alpha+1) C$ for all $x \leqq$ $x_{1}$. Suppose first that $\alpha \leqq 0$. Define $Q_{1}(x)=\alpha C, a \leqq x \leqq x_{1}$, and $Q_{2}=$ $Q-Q_{1}$. Then $-Q_{1} \leqq C$ and for $a \leqq x \leqq x_{1}, \int_{a}^{x} Q_{2} d \mu$ satisfies

$$
\begin{aligned}
-C \leqq \int_{a}^{x} Q d \mu \leqq \int_{a}^{x} Q_{2} d \mu & =\int_{a}^{x} Q d \mu-\alpha C \mu([a, x]) \\
& \leqq(\alpha+1) C-\alpha C=C .
\end{aligned}
$$

Now suppose that $\alpha>0$. Let $m$ be the integer such that $m<\alpha \leqq$ $m+1$. For $j=0,1, \cdots, m$, set 


$$
a_{j}=\max \left\{x<x_{1}: \int_{a}^{x} Q d \mu=j C\right\} .
$$

Set $a_{m+1}=x_{1}$. Then $a \leqq a_{0}<a_{1}<\cdots<a_{m+1}$. Choose $b_{j}>a_{l}, j=0$, $1, \cdots, m-1$ so that

$$
\int_{a_{1}}^{b_{1}} Q^{+} d \mu=C
$$

and $b_{m}$ so that $\int_{a_{m}}^{b_{m}} Q^{+} d \mu=(\alpha-m) C$. Here $Q^{+}=\max \{Q, 0\}$ as usual. Note that $a_{\jmath}<b_{\jmath} \leqq a_{\jmath+1}$ and that $Q^{-}=Q^{+}-Q$ satisfies $\int_{a_{1}}^{b_{1}} Q^{-} d \mu<C$.

Define $Q_{1}=Q^{+}$on $\bigcup_{j=0}^{m}\left[a_{j}, b_{l}\right], Q_{1}=0$ otherwise, and $Q_{2}=$ $Q-Q_{1}$. Then $Q_{1} \geqq 0$ so we need only verify that $Q_{2}$ has the required property. If $x<a_{0}$, then $Q_{2}=Q$ where

$$
-C \leqq \int_{a}^{x} Q d \mu \leqq C
$$

The last inequality follows from the fact that $\int_{a}^{a_{0}} Q d \mu=0$. If $a_{1} \leqq x \leqq$ $b_{\jmath}, j=0,1, \cdots, m$, then $\int_{a}^{x} Q_{2} d \mu=\int_{a_{1}}^{x} Q_{2} d \mu$ and

$$
-C<\int_{a_{1}}^{b_{1}} Q_{2} d \mu \leqq \int_{a_{1}}^{x} Q_{2} d \mu \leqq 0
$$

since $Q_{2}=Q-Q^{+}$on $\left[a_{1}, b_{1}\right]$.

If $b_{\jmath} \leqq x \leqq a_{\jmath+1}, j \leqq m-1$, then

$$
\int_{a}^{x} Q_{2} d \mu=\int_{a}^{x} Q d \mu-(j+1) C \leqq(j+2) C-(j+1) C=C .
$$

Similarly, if $b_{m} \leqq x \leqq a_{m+1}$, then $\int_{a}^{x} Q_{2} d \mu=\int_{a}^{x} Q d \mu-\alpha C \leqq C$.

Finally, for $b_{\jmath} \leqq x \leqq a_{\jmath+1}$,

$$
\int_{a}^{x} Q_{2} d \mu=\int_{a_{1}}^{b_{1}} Q_{2} d \mu+\int_{b_{1}}^{x} Q d \mu \geqq-C
$$

since otherwise $\int_{a}^{x} Q d \mu=j C$ for some $x>a_{j}$. Thus the decomposition 
$Q=Q_{1}+Q_{2}$ has the property (ii) with $C_{1}=C_{2}=C$ and the proof of the lemma is complete.

We now conclude the proof of Theorem 3. Applying the lemma with $Q=q w^{2}$ and $d \mu=w^{-1} d t$, we have that $q=q_{1}+q_{2}$ where $-q_{1} w^{2} \leqq$ $C_{1}$ and $\left|\int_{a}^{x} q_{2} w d t\right| \leqq C_{2}$. This is (iii) and (iv) of Theorem 1 with $b=0$. Thus Theorem 3 is a consequence of Theorem 1 .

6. A perturbation result. There have been some investigations recently into whether the limit-point property for a differential expression is preserved under the addition of a term $Q y$ where the integral of $Q$ is small in some sense (generally $Q$ is assumed in $L_{r}(a, \infty)$ for some $r \geqq 1)$. In this direction we offer the following extension of a result of Patula and Wong [9].

THEOREM 4. Suppose that (1.1) satisfies the hypotheses of Theorem 1 with $b=1$ and that $p$ is bounded away from 0 . Suppose that $Q$ is a locally integrable function such that for some $r \geqq 1$ and all $x \geqq a$,

$$
\int_{x}^{x+1}|Q|^{r} d t \leqq K
$$

Then $-\left(p y^{\prime}\right)^{\prime}+(q+Q) y$ satisfies the hypotheses of Theorem 1 with $b=1$.

Proof. We may assume that $Q$ is real-valued. (In fact the result is true if $Q$ is replaced by $\operatorname{Re} Q$ in (6.1).) It then follows from (6.1) and Schwarz's inequality that $\int_{x}^{x+d} Q d t \geqq-K^{1 / r}$ for each $x \geqq a$ and each positive $d \leqq 1$. Thus by the lemma of $\S 5$ with $d \mu=d t, Q=Q_{1}+Q_{2}$ with $-Q_{1} \leqq C_{1}$ and $\left|\int_{a}^{x} Q_{2} d t\right| \leqq C_{2}$ for all $x \geqq a$. Then $-\left(q_{1}+Q_{1}\right) \leqq$ $-q_{1}+C_{1}$ and

$$
\left|\int_{a}^{x} q_{2}+Q_{2} d t\right| \leqq\left|\int_{a}^{x} q_{2} d t\right|+C_{2}
$$

Let $w$ be the nonnegative locally absolutely continuous function of Theorem 1 for (1.1). If $w$ is bounded then it is clear from the previous paragraph that the hypotheses of Theorem 1 are also satisfied for the new expression with the same choice for $w$.

Suppose, then, that $w$ is not bounded. Set

$$
W(x)=w(x) /\left(1+\int_{a}^{x} w p^{-1 / 2} d t\right)
$$


We assert that $W$ is bounded and has the other necessary properties. Suppose for some $x_{1}$ that $w\left(x_{1}\right)>1$. Set $x_{0}=\max \left\{x<x_{1}\right.$ : $w(x) \leqq 1\}$. Then on $\left[x_{0}, x_{1}\right],\left|w^{\prime}\right| \leqq K_{1} w p^{-1 / 2}$ so that

$$
w\left(x_{1}\right) \leqq 1+K_{1} \int_{a}^{x_{1}} w p^{-1 / 2} d t .
$$

This inequality also holds if $w\left(x_{1}\right) \leqq 1$. Thus $\lim \sup W \leqq K_{1}$.

We now verify the hypotheses of Theorem 1 . For $x \geqq a$,

$$
W^{\prime}(x)=w^{\prime}(x) /\left(1+\int_{a}^{x} w p^{-1 / 2} d t\right)-\{W(x)\}^{2}\{p(x)\}^{-1 / 2}
$$

so that $p^{1 / 2} W^{\prime}$ is bounded. Also

$$
\int_{a}^{x} W p^{-1 / 2} d t=\log \left(1+\int_{a}^{x} w p^{-1 / 2} d t\right)
$$

so that this integral tends to infinity with $x$.

Finally, $W \leqq w$ so that

$$
-\left(q_{1}+Q_{1}\right) W^{2} \leqq K_{2}-Q_{1} W^{2} \leqq K_{2}+C_{1}(\max W)^{2}
$$

and

$$
\{p(x)\}^{-1 / 2} W(x)\left|\int_{a}^{x} q_{2}+Q_{2} d t\right| \leqq K_{3}+C_{2} \max \left\{p^{-1 / 2} W\right\}
$$

Thus the hypotheses of Theorem 1 are satisfied for $-\left(p y^{\prime}\right)^{\prime}+(q+Q) y$ and the proof is complete.

\section{REFERENCES}

1. F. V Atkinson and W. D. Evans, On solutions of a differential equation which are not of integrable square, Math Z., 127 (1972), 323-332.

2. I. Brinck, Self-adjointness and spectra of Sturm-Liouville operators, Math. Scand., 7 (1959), 219-239.

3. E. A. Coddington and N. Levinson, Theory of Ordinary Differential Equations, McGraw-Hill, New York, 1955.

4. M. S. P. Eastham, On a limit-point method of Hartman, Bull. London Math. Soc., 4 (1972), 340-344.

5. P. Hartman and A. Wintner, A criterion for the non-degeneracy of the wave equation, Amer. J. Math., 71 (1949), 206-213.

6. R. M. Kauffman, Polynomials and the limit point condition, Trans. Amer. Math. Soc., 201 (1975), $347-366$. 
7. I. Knowles, A limit-point criterion for a second-order linear differential operator, J. London Math. Soc., 8 (1974), 719-727.

8. N. Levinson, Criteria for the limit-point case for second order linear differential operators, Časopis Pěst. Mat., 74 (1949), 17-20.

9. W. T. Patula and J. S. W. Wong, An $L^{p}$-analogue of the Weyl's alternative, Math. Ann., 197 (1972), 9-27.

Received April 24, 1975 and in revised form April 12, 1976.

UNIVERSITY OF DUNDEE

AND

Western Washington State College 




\section{Pacific Journal of Mathematics}

\section{Vol. 66, No. $1 \quad$ November, 1976}

Helen Elizabeth. Adams, Factorization-prime ideals in integral domains ............ Patrick Robert Ahern and Robert Bruce Schneider, The boundary behavior of Henkin's kernel.

Daniel D. Anderson, Jacob R. Matijevic and Warren Douglas Nichols, The Krull

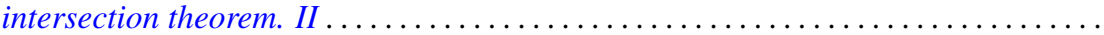

Efraim Pacillas Armendariz, On semiprime P.I.-algebras over commutative regular

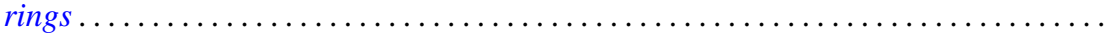

Robert H. Bird and Charles John Parry, Integral bases for bicyclic biquadratic fields

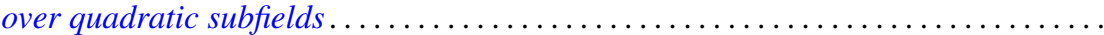

Tae Ho Choe and Young Hee Hong, Extensions of completely regular ordered

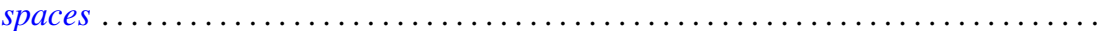

John Dauns, Generalized monoform and quasi injective modules ...............

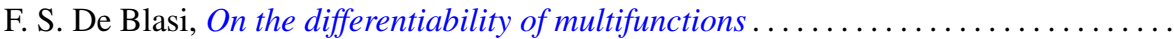

Paul M. Eakin, Jr. and Avinash Madhav Sathaye, R-endomorphisms of $R[[X]]$ are

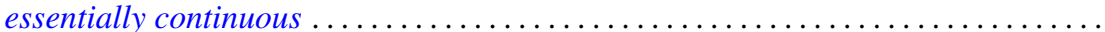

Larry Quin Eifler, Open mapping theorems for probability measures on metric

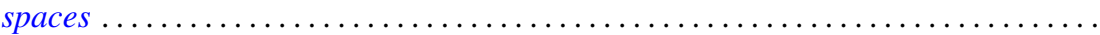

Garret J. Etgen and James Pawlowski, Oscillation criteria for second order self adjoint

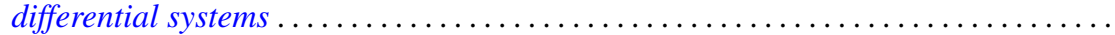

Ronald Fintushel, Local $S^{1}$ actions on 3-manifolds .

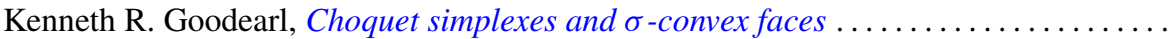

John R. Graef, Some nonoscillation criteria for higher order nonlinear differential

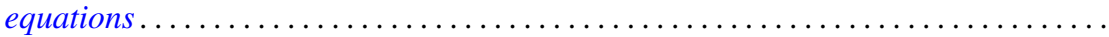

Charles Henry Heiberg, Norms of powers of absolutely convergent Fourier series: an example.

Les Andrew Karlovitz, Existence of fixed points of nonexpansive mappings in a space

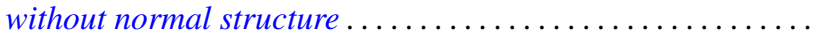

Gangaram S. Ladde, Systems of functional differential inequalities and functional

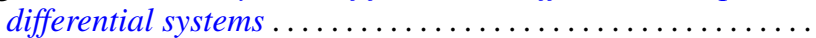

Joseph Michael Lambert, Conditions for simultaneous approximation and interpolation with norm preservation in $C[a, b]$.

Ernest Paul Lane, Insertion of a continuous function.

Robert F. Lax, Weierstrass points of products of Riemann surfaces .

Dan McCord, An estimate of the Nielsen number and an example concerning the Lefschetz fixed point theorem...

Paul Milnes and John Sydney Pym, Counterexample in the theory of continuous functions on topological groups...

Peter Johanna I. M. De Paepe, Homomorphism spaces of algebras of holomorphic functions

Judith Ann Palagallo, A representation of additive functionals on $L^{p}$-spaces,

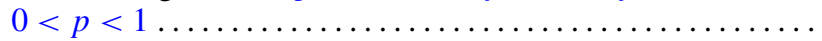

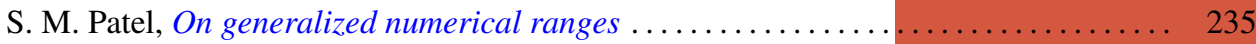

Thomas Thornton Read, A limit-point criterion for expressions with oscillatory

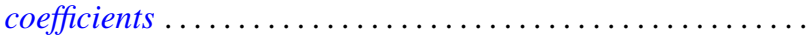

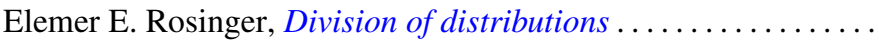

Peter S. Shoenfeld, Highly proximal and generalized almost finite

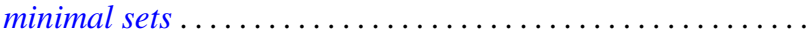

R. Sirois-Dumais and Stephen Willard, Quotient-universal sequential spaces

Robert Charles Thompson, Convex and concave functions of singular values of matrix sums....

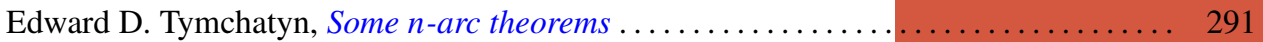

